\title{
Synaptonemal complex morphogenesis and sister-chromatid cohesion require Mek1-dependent phosphorylation of a meiotic chromosomal protein
}

\author{
Julie M. Bailis ${ }^{2}$ and G. Shirleen Roeder ${ }^{1-4}$ \\ ${ }^{1}$ Howard Hughes Medical Institute, ${ }^{2}$ Department of Molecular, Cellular and Developmental Biology, ${ }^{3}$ Department \\ of Genetics, Yale University, N ew Haven, Connecticut 06520-8103 USA
}

\begin{abstract}
Development of yeast meiotic chromosome cores into full-length synaptonemal complexes requires the MEK 1 gene product, a meiosis-specific protein kinase homolog. The Mek1 protein associates with meiotic chromosomes and colocalizes with the Redl protein, which is a component of meiotic chromosome cores. Mek1 and Red1 interact physically in meiotic cells, as demonstrated by coimmunoprecipitation and the two-hybrid protein system. Hopl, another protein associated with meiotic chromosome cores, also interacts with Mek1 but only in the presence of Red1. Red1 displays Mek1-dependent phosphorylation, both in vitro and in vivo, and Mek1 kinase activity is necessary for Mek1 function in vivo. Fluorescent in situ hybridization analysis indicates that Mek1-mediated phosphorylation of Red1 is required for meiotic sister-chromatid cohesion, raising the possibility that cohesion is regulated by protein phosphorylation.
\end{abstract}

[Key Words: Chromosome cores; lateral elements; meiosis; phosphorylation; sister-chromatid cohesion; synaptonemal complex]

Received June 30, 1998; revised version accepted September 23, 1998.

M eiosis generates haploid gametes from dipl oid parental cells through two rounds of chromosome segregation following a single round of DNA replication. Specialized interactions between homologous chromosomes-pairing, synapsis, and recombination-occur during mei otic prophase and are essential for the reductional segregation of chromosomes at meiosis I. Each homolog consists of two sister chromatids that remain associated throughout the first meiotic division.

During meiotic prophase, homologous chromosomes become physically associated along their lengths through a protei naceous structure called the synaptonemal complex (SC; for review, see Roeder 1997). Early in SC assembly, each pair of sister chromatids develops a common protein core, referred to as an axial element. As chromosomes synapse, proteins assemble between the axial el ements to form the central region of the SC. Axial elements are called lateral elements in the context of mature complex. The SC extends al ong the full lengths of chromosomes at pachytene and then disassembles in dipl otene.

In Saccharomyces cerevisiae, several proteins associated with the SC have been characterized. The Zipl pro-

${ }^{4}$ Corresponding author.

E-MAIL shirleen.roeder@yale.edu; FAX (203) 432-3263. tein localizes along the lengths of synapsed chromosomes and is a component of the SC central region (Sym et al . 1993). The Hopl and Redl proteins are components of axial and lateral el ements (Hollingsworth et al. 1990; Smith and Roeder 1997). red 1 mutants fail to form axial elements or SC (Rockmill and Roeder 1990); hop1 mutants assemble axial elements, but these do not synapse (Hollingsworth and Byers 1989; Loidl et al. 1994). The RED 1 and HOP1 genes interact in genetic assays (Hollingsworth and Johnson 1993; Friedman et al . 1994; Hollingsworth and Ponte 1997), and the Redl and Hopl proteins colocalize on meiotic chromosomes (Smith and Roeder 1997), suggesting that these gene products participate in the same pathway or process.

Characterization of meiotic mutants in budding yeast and other organisms has provided information about the functions of the SC. The results suggest that the primary function of the SC central region is to regulate the distribution of meiotic crossovers along and among chromosomes (Sym and Roeder 1994). The functions of axial and lateral elements are less well defined, but there is some indication that these structures play important roles in mei otic chromosome segregation. Proper reductional segregation at meiosis I depends on the formation of chiasmata, which are chromatin bridges between homologous chromosomes that correspond in position to the sites of genetic crossovers. Chiasma function is pre- 
sumed to depend on sister-chromatid cohesion; homologs are held together at crossover sites because of cohesion between sister chromatids in regions distal to chiasmata (M iyazaki and Orr-Weaver 1994). The yeast red1 mutant is apparently defective in sisterchromatid cohesion and/or chiasma function because chromosomes that have undergone crossing-over nevertheless fail to segregate correctly (Rockmill and Roeder 1990). The hamster Cor1 protein is another candidate for a lateral element protein that is involved in meiotic sister-chromatid cohesion, as Corl localizes to chromosome axes through metaphase I (M oens and Spyropoulos 1995). In fission yeast, the Rec8 protein is necessary both for formation of linear elements (lateral element-like structures) and for meiotic sister-chromatid cohesion (Molnar et al. 1995). Rec8 is homologous to the budding yeast $\mathrm{Mcd} 1 / \mathrm{Sccl}$ protein, which participates in sister-chromatid cohesion during mitosis (Guacci et al. 1997; Michaelis et al. 1997). Thus, sisterchromatid cohesion in mitosis and meiosis may be mechanistically related.

Despite progress in defining the role of SC proteins in sister-chromatid cohesion and meiotic chromosome development, little is known about how these functions are regulated. There is evidence, however, that protein phosphorylation is important. The rat SCP3 protein, a component of lateral elements, undergoes a change in phosphorylation during meiotic prophase (Lammers et al. 1994). The yeast Red1 protein interacts with a phosphatase in the two-hybrid-protein system (Tu et al. 1996). Additionally, the mouse Atm and Atr protein kinases, and mammalian homologs of the fission yeast Chk1 kinase, have been localized to meiotic chromosomes (Keegan et al. 1996; Flaggs et al. 1997).

The S. cerevisiae MEK1/MRE4 gene encodes a protein kinase homolog that is expressed specifically in meiosis. In a mek1 null mutant, meiotic recombination is reduced to $\sim 20 \%$ of the wild-type level (Rockmill and Roeder 1991; Leem and Ogawa 1992). The mek 1 mutant forms multiple, short stretches of SC rather than fulllength complexes (Rockmill and Roeder 1991). Genetic analysis indicates that MEK1 is in the same epistasis group as RED1 and HOP1 (Rockmill and Roeder 1991), and overproduction studies suggest that Mek1, Red1, and Hopl interact with a defined stoichiometry (Hollingsworth and Ponte 1997; J.M. Bailis and G.S. Roeder, unpubl.). A Mek1- $\beta$-galactosidase fusion protein localizes to the nucleus (Burns et al. 1994), raising the possibility that Mek 1 associates with meiotic chromosomes. Although it has been inferred from genetic data that M ek1 interacts with Red1 and Hopl (Hollingsworth and Ponte 1997), the nature and function of these interactions have not been elucidated. Here, we demonstrate that the wild-type Mek1 protein functions as a kinase that phosphorylates Red1 and associates with both Red1 and Hopl. We propose that interaction of Mek 1 with axial and lateral el ement components promotes SC morphogenesis and establishes meiotic sister-chromatid cohesion.

\section{Results}

Mek1 associates with meiotic chromosomes

To gain insight into the function of M ek1, anti-Mek1 antibodies were raised and used to localize the protein within mei otic cells. M eiotic chromosomes that are surface spread and stained with anti-Mek1 antibodies display numerous foci, indicating that Mek1 associates with chromosomes (Fig. 1). This staining pattern is specific for Mek1, as no antibody staining is observed in spread nuclei from a mek1 null mutant (JM 82; data not shown).

The timing and pattern of Mek1 localization was assessed by staining meiotic chromosomes from wild-type cells (BR2495) with antibodies to both Mek1 and Zipl. Chromosomal DNA was stained with 4'-6-diamino-2phenolindole (DAPI). Zipl local ization indicates regions where chromosomes are synapsed (Sym et al. 1993; Smith and Roeder 1997). In early zygotene, spread chromosomes display numerous Zipl-staining foci, corresponding to sites of synapsis initiation. Later in zygotene, when chromosomes are partially synapsed, both Zipl dots and linear stretches of Zipl stai ning are apparent. At pachytene, chromosomes are fully condensed and Zipl local izes continuously al ong the length of each pair of homologous chromosomes.

A comparison of the M ek1 and Zipl localization patterns reveals that Mek1 foci first appear on chromosomes in early zygotene, reach a maximum number at pachytene, and disappear from chromosomes sometime after pachytene (Fig. 2). In early zygotene, $\sim 5 \%-10 \%$ of spread nuclei that display Zipl foci fail to exhibit Mek1 staining. However, all early zygotene nuclei that display M ek1 foci al so exhibit Zip1 foci (category 1, $24 \pm 8$ M ek1 foci per nucleus; Fig. 1A-C). Nuclei that display Ziplstaining dots and linear stretches contain an average of $30( \pm 8)$ M ek1 foci per nucleus (category 2; Fig. 1D-F,J-L). This category peaks at two points (Fig. 2), one before pachytene and the other after pachytene. The early peak is likely to represent nuclei in which chromosomes are partially synapsed (late zygotene); the later peak probably represents nuclei undergoing SC disassembly (diplotene). At pachytene (category 3), there are 40 ( \pm 4 ) M ek 1 foci per nucleus (Fig. 1G-I). Surprisingly, Mek1 chromosomal localization persists in spread nuclei from late time points in which little or no Zipl is detected (category 4, $17 \pm 5$ Mek 1 foci per nucleus; Fig. 1M-O; data not shown).

To determine whether $M$ ek 1 remains associated with chromosomes during the meiosis I division, spread chromosomes prepared at late time points in meiosis were double labeled with antibodies to Mek1 and tubulin. Mek1 foci are observed in spread nuclei that contain a short meiosis I spindle (Fig. 1M-O) but not in nuclei that have completed meiosis I or at meiosis II (data not shown). These results indicate that Mekl gradually dissociates from chromosomes during the period from diplotene through metaphase I.

To determine whether M ek1 kinase activity is impor- 

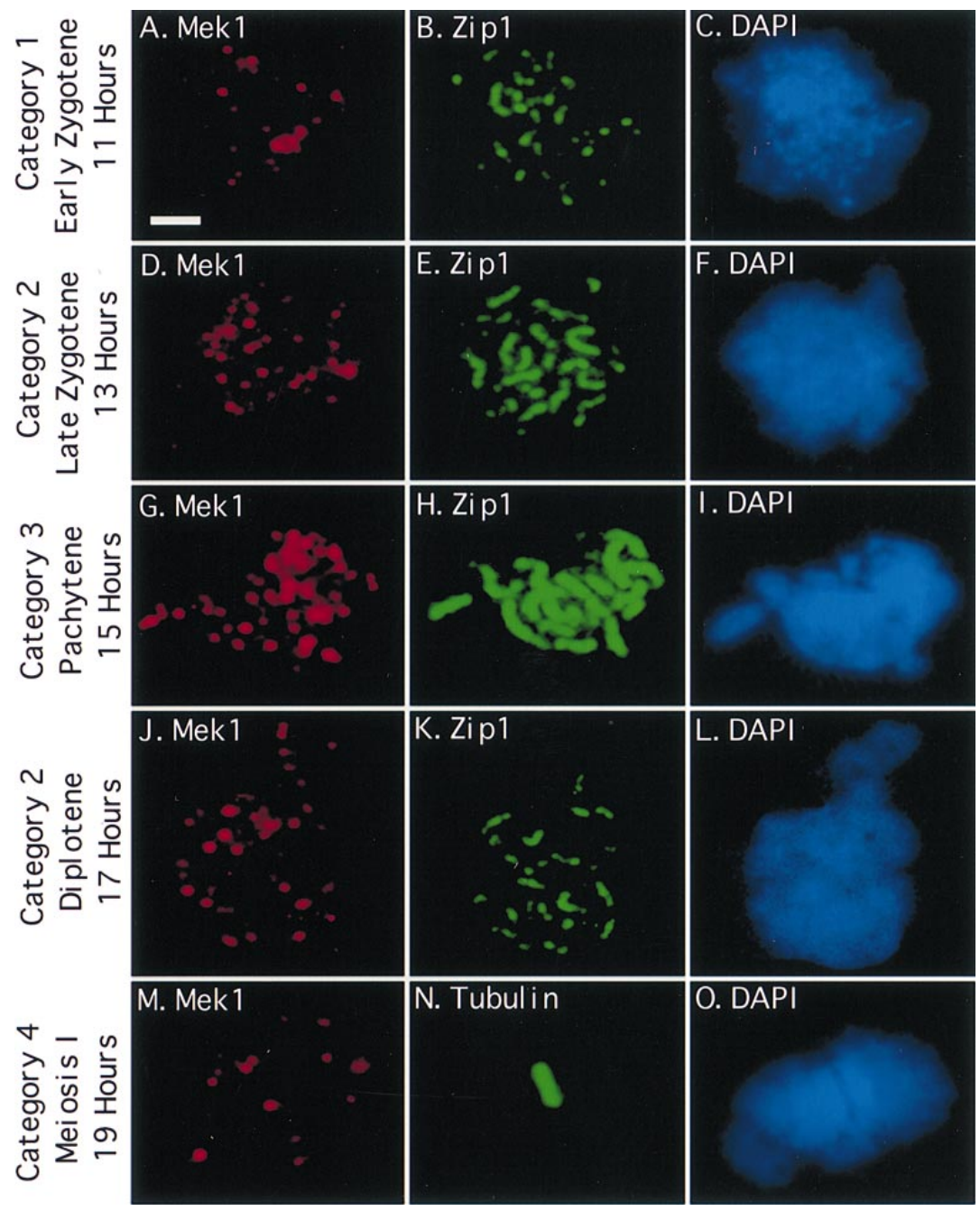

Figure 1. Mek1 localizes to chromosomes during and after synapsis. Spread meiotic chromosomes from wild type (BR2495) were stained with antibodies to Mek1 (A,D,G,J,M), antibodies to Zipl (B,E,H,K) or tubulin (N), and DAPI $(C, F, I, L, O)$. Categories of Mek1 staining are indicated at left (see text for explanation). Scale bar, $2 \mu \mathrm{m}$. tant for its localization, a conserved residue in the M ek1 kinase domain was changed to create the mek1-D290A mutation. The conserved aspartate resi due (D290) is presumed to be the kinase catalytic site, and mutation of this residue in other protein kinases abol ishes or significantly decreases protein kinase activity (Zhou and Elledge 1993; Sun et al. 1996). The spore viability of the mek1-D290A mutant is equivalent to that of the mek $1:$ :LYS2 null mutant (18.6\% vs. $19.2 \%, 100$ tetrads analyzed per strain). The mutant Mek1-D290A protein does, however, localize to chromosomes (Fig. 3M-P); M ek1 therefore local izes to chromosomes independently of its presumed kinase activity. Furthermore, the pattern and timing of localization of the mutant protein are similar to those of the wild-type Mek 1 protein.

The Mek1- $\beta$-galactosidase fusion protein (Mek1- $\beta$ gal) shown previously to be nuclear (Burns et al. 1994) also localizes to chromosomes with a staining pattern that is indistinguishable from that of the authentic Mek1 protein (Fig. 3E-H). This fusion protein contains only the first 63 amino acids of Mek 1 and lacks the entire $M$ ek 1 kinase domain, indicating that this region of Mek1 is sufficient for proper localization.
Mek1 colocalizes with Red1 and transiently with Hop1

To test whether Mek1 colocalizes with Red1 and/or Hop1, spread nuclei prepared from several time points in meiosis were double labeled with antibodies to Mek1 and Red1 or M ek1 and Hopl. The Red1 staining pattern is punctate in early prophase and then becomes somewhat continuous by pachytene (Smith and Roeder 1997). Hopl foci reach a maximum number in early prophase; by late pachytene, Hopl is largely dissociated from chromosomes (Smith and Roeder 1997). Red1 and Hopl display extensive colocalization early in prophase, but the number of overlapping foci decreases as cells progress through prophase, and Hopl dissociates from chromosomes while Red1 accumulates (Smith and Roeder 1997). In the BR2495 strain background, the dissociation of Hop1 from chromosomes correl ates with the association of Zipl with chromosomes and therefore with the formation of mature SC (Smith and Roeder 1997).

At least $95 \%$ of $\mathrm{Mek} 1$ foci in spread nuclei that are in early zygotene through pachytene colocalize with areas of Redl staining (Fig. 3A-D). However, there are usually 


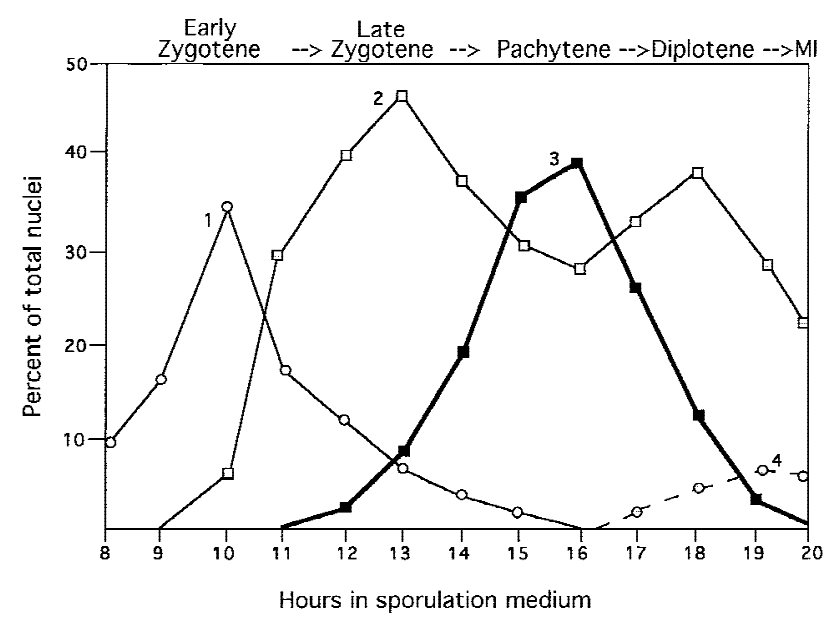

Figure 2. Time course analysis of M ek 1 and Zipl local ization. Spread nuclei from wild type (BR2495) were prepared hourly during meiosis and double stained with antibodies to M ek 1 and Zip1. The frequencies of the four categories of Mek1 localization are indicated; at least 100 nuclei were examined per time point. (Category 1) Early zygotene, punctate Zipl staining; (category 2) Zip1 dots and linear stretches; first peak, late zygotene; second peak, di plotene; (category 3) pachytene, continuous Zip1 staining; (category 4) meiosis I (MI). The average number of Mek1 foci per nucleus in categories $1-4$ is $24,30,40$ and 17 , respectively.

regi ons of Red1 staining that do not contain M ek1. After pachytene, Mek1 foci are present even though Red1 has mostly dissociated from chromosomes (Smith and Roeder 1997; data not shown). The Mek1- $\beta$-gal protein also colocalizes with Red1 (Fig. 3E-H), reinforcing the conclusion that the localization pattern of the Mek1- $\beta$ gal protein is similar to that of wild-type Mek1.

In contrast, very few Mek1 foci colocalize with Hop1 foci on spread chromosomes from wild type (Fig. 3I-L). At $11 \mathrm{hr}$ (early zygotene), an average of $8 \%$ (range, 5\%$27 \%$ ) of M ek1 foci overlap with Hopl foci. At $13 \mathrm{hr}$ (late zygotene), an average of $36 \%$ (range, $23 \%-52 \%$ ) of M ek 1 foci overlap with Hop1, whereas an average of $18 \%$ overlap (range, 10\%-25\%) is observed at $14 \mathrm{hr}$ (early pachytene). Fortuitous overlap of Mek1 with Hopl is estimated at $\sim 6 \%$ or less (Gasior et al. 1998). The amount of overlap between Mek1 and Hopl varies over time, as Hop1 is already abundant on chromosomes when M ek1 is first detected, and Hopl is largely dissociated from chromosomes when Mek1 foci are at a maximum. This could be explained if either Mek1 and Hopl localize to the same foci, but at different times, or if Hopl associates with some of the Red1-staining foci, while Mek1 associates with other sites of Red1 localization.

Genetic assays support the view that Mek1 and Hopl interact (Hollingsworth and Ponte 1997; J.M. Bailis and G.S. Roeder, unpubl.), suggesting either that Mek1 and Hopl interact transiently or Mek1 replaces Hopl as Hopl dissociates from chromosomes. To test whether Mek1 kinase activity affects Hopl dissociation from chromosomes, spread chromosomes from the mek1D290A mutant were double labeled with antibodies to Mek1 and Hopl (Fig. 3M-P). Hopl associates with chromosomes on time in the mutant, but Hopl dissociation from chromosomes appears to be delayed compared to wild type. In early zygotene (11 hr), similar numbers of Hopl foci are observed in wild type and mutant (average of 61 foci per nucleus both in wild type and mutant). However, by early pachytene (14 hr), the average number
Figure 3. Mek1 localization pattern compared to Red1 and Hopl. A wild-type spread nucleus (BR2495) was stained with antibodies to Mek1 (A) and Red1 (B) and with DAPI (D); (C) fusion of $A$ and $B$. A spread nucleus from the mek1-lacZ mutant (JM 198) was stained with antibodies to $\beta$-gal actosidase (E) and Red $1(F)$ and with DAPI $(H) ;(G)$ fusion of $E$ and F. A wild-type spread nucleus (BR2495) was stained with antibodies to Mek1 (I) and Hopl (J) and with DAPI (L); (K) fusion of I and J. A spread nucleus from the mek1-D290A mutant (JM 191) was stained with antibodies to M ek1 (M) and Hopl (N) and with DAPI (P); $(\mathrm{O})$ fusion of $M$ and $N$. All nuclei are from a 13-hr time point. A reas of yellow in the merged images indicate overlap. Scale bar, $2 \mu \mathrm{m}$.
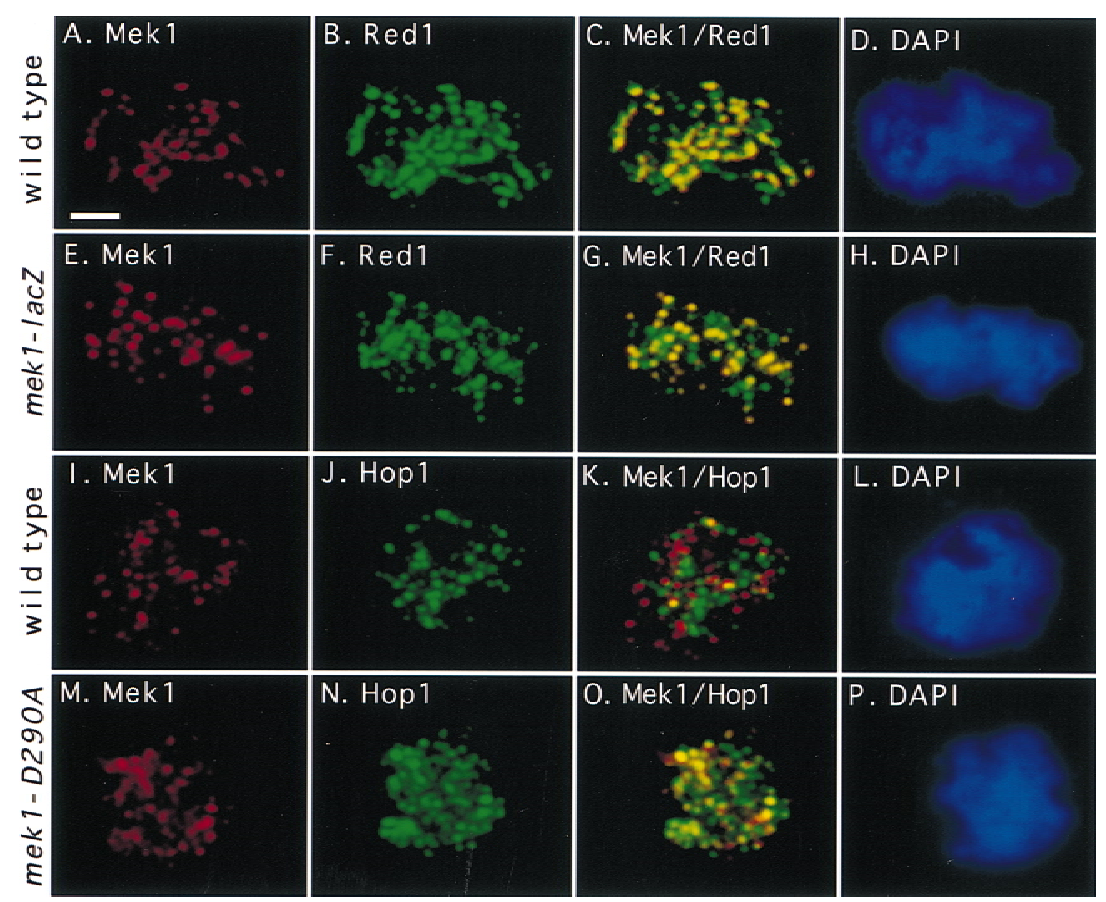

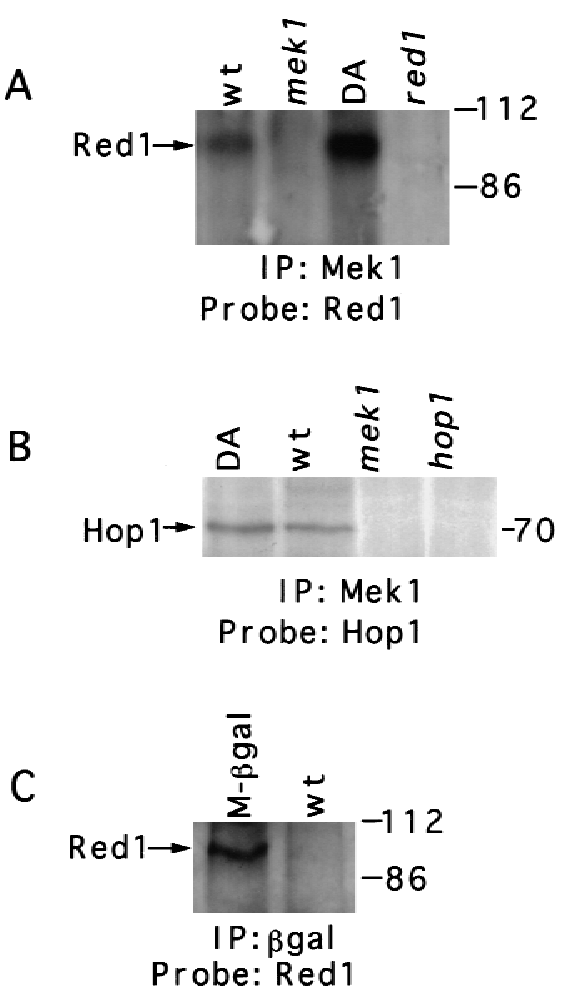
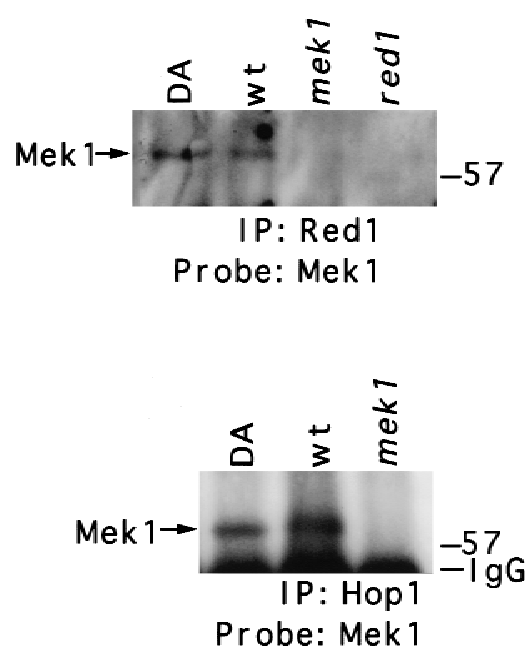

Probe: Mek 1
Figure 4. Association of Mek 1 with Red1 and Hopl in meiotic cells. (A) Anti-Mek1 antibodies coprecipitate Red1, and antiRed1 antibodies coprecipitate Mek1. (B) Coimmunoprecipitation of $M e k 1$ and Hop1. (C) Red1 coprecipitates with the Mek1- $\beta$-gal protein, which contains just the amino-terminal region of Mekl. Proteins were immunoprecipitated from $1 \mathrm{li}$ ter of cells that had been sporulated for 13 $\mathrm{hr}$ and then analyzed by SDS-PAGE and immunoblotting. Antibodies used for immunoprecipitation (IP) and for immunoblotting (Probe) are indicated on the bottom of each blot. When antibodies to Hopl are used to coimmunoprecipitate M ek1, as in $B$, Mekl is not detected in the hopl mutant (data not shown). Molecular mass markers are shown at the right of each blot. (IgG) Immunogl obulin G. Strains used are wild type (BR2495), mek1-D290A (JM 191), mek 1::LYS2 (JM 82), red1::U RA3 (MY231), hop1::TRP1 (BR2498), and mek1-lacZ (JM 198). (wt) Wild type; (DA) mek1D290A; (M- $\beta$ gal) M ek1- $\beta$-gal actosi dase. of Hopl foci per nucleus is 35 in wild type but somewhat higher (44 foci per nucleus) in the mutant. The delay in the dissociation of Hopl from chromosomes is associated with an increased colocalization of Mek1 with Hopl. In early pachytene, $80 \%$ of M ek 1 foci overlap with Hop1 in mek1-D290A, whereas just 18\% of M ek1 foci on wild-type spread chromosomes overlap with Hop1 foci. This increased col ocal ization is not due to a del ay in meiosis or to an increase in the number of Mekl foci (data not shown). These results argue that Mek1 and Hopl localize transiently to the same chromosomal foci and suggest that Mek1 kinase function promotes Hop1 dissociation from meiotic chromosomes.

\section{Mek1 chromosomal localization depends on Red1 and Hopl}

To determine whether Red1 and/or Hopl are required for M ek 1 local ization to chromosomes, anti-M ekl antibodies were used to stain spread chromosomes prepared from red 1 and hopl mutants. Both M ek 1 and the M ek1$\beta$-gal protein fail to localize to chromosomes in a red1 null mutant and localize to only $\sim 5 \%$ of spread nuclei from a hop1 null mutant (strains M Y231, JM 93, BR2498, and JM 88; data not shown). When whole meiotic cells from the red1 or hopl mutant are stained with antibodies to $M$ ek 1 , M ek1 staining is detected in the cytoplasm rather than in the nucleus (data not shown).

The inability to detect Mek1 chromosomal localization in red 1 and hopl strains is not due to instability of the Mek1 protein. Anti-Mek1 immunoblots of whole mei otic cell extracts prepared from red1 or hopl mutants detect the M ek 1 protein in amounts similar to wild type (data not shown). In red 1 or hopl mutants contai ning the M ek1- $\beta$-gal protein, the level of $\beta$-galactosidase activity is similar to that of an otherwise wild-type (i.e., RED 1 HOP1) strain [72.8 units of $\beta$-galactosidase in wild type (JM 198), 76.2 units in red1 (JM 93), and 74.6 units in hop1 (JM 88)].

These data indicate that Red1 and Hopl are required for Mek1 localization to the nucleus and to chromosomes. Taken together with the requirement of Red1 for Hopl localization (Smith and Roeder 1997), these observations imply a specific order of protein association: Red1, then Hopl, and finally M ek1.

\section{Red1 and Hopl coimmunoprecipitate with Mek1}

Immunoprecipitation experiments were carried out to determine whether M ek 1 physically interacts with Red1 and Hopl. Extracts of sporulating cells were prepared (see Materials and M ethods), and M ek1, Red1, or Hopl was immunoprecipitated with appropriate antibodies and anal yzed by SDS-PAGE and immunoblotting. M ek 1 and Red1 coprecipitate from wild type (BR2495) and from mek1-D290A (JM 191) (Fig. 4A). Mek1 and Hop1 also coimmunoprecipitate from these strains (Fig. 4B). Other proteins coimmunopreci pitate with M ek 1 as well, as determined by silver staining of protein gels (data not shown). None of these proteins, including Redl and Hopl, are detected in immunoprecipitates from a mek1 null mutant or from a wild-type extract immunoprecipitated using preimmune serum (data not shown).

Colocalization of the Mek1- $\beta$-gal protein with Red1 
raised the possibility that the amino-terminal region of M ek1 interacts with Red1. To test this possibility, antibodies to $\beta$-galactosidase were used to immunoprecipitate Mek 1 and associated proteins from meiotic cell extracts prepared from a strain in which the Mek1- $\beta$-gal protein is the only source of Mek1 (JM 198). Red1 coprecipitates with Mek1- $\beta$-gal (Fig. 4C), as does Hopl (data not shown).

\section{Mek1 interacts with Hopl in the presence of Red1}

The two-hybrid protein system was used to test for a direct interaction of M ek 1 with Redl or Hopl. M ek1 and Red1 do interact in the two-hybrid system, and the first 63 amino acids of Mek1 are sufficient to establish this interaction (Table 1). However, an interaction between Mek1 and Hopl is not detected in vegetative cells.

If the interaction between Mek1 and Hopl requires Red1, then a M ek1-Hopl interaction should be detected in meiotic cells (where Red1 is expressed) or in vegetative cells producing Red1. This is indeed the case. A strong interaction between M ek 1 and Hopl is detected in meiotic cells from wild type, but not from a red1 mutant (Table 1). An interaction between Mek1 and Hopl is also observed in vegetative cells overproducing Red1 (Table1). The greater intensity of interactions observed in mei otic cells compared to vegetative cells may be due to meiosis-specific protein modification.

\section{Mek1 has protein kinase activity}

To test whether the M ek1 homol ogy to serine/threonine protein kinases is significant for its function, M ek1 protein kinase activity was examined. M ek 1 and associated proteins were immunoprecipitated from meiotic cell extracts, and Mek1 phosphorylation capability was then assayed in vitro.

When anti-M ek1 immunoprecipitates are incubated in vitro with $\left[\gamma^{-32}\right.$ P]ATP, a phosphorylated band of the ap- proximate molecular mass predicted for Mek1 ( 60 kD) is observed; this band comigrates with the M ek 1 protein as determined by immunoblotting (Fig. 5A). Evidence that the phosphorylated band is indeed Mek 1 was obtained using a Mek1-green fluorescent protein (Mek1GFP) fusion as the only source of Mek1. The 75-kD Mek1-GFP al so undergoes phosphorylation in vitro (Fig. $5 \mathrm{~A}$ ). Other phosphorylated bands in the autoradiograph are likely to represent other proteins that coprecipitate with M ek1. In contrast to the wild-type Mek1 protein, the mutant Mek1-D290A protein displays little or no phosphorylation in vitro, although immunoblotting indicates that the amount of protein present is similar to that in wild type (Fig. 5A).

A gel mobility shift assay was used to examine whether the Mek1 protein is itself phosphorylated in vivo. Mek1 that is immunoprecipitated from wild-type meiotic cell extracts is present in two mobility forms (Fig. 5B). The slower migrating form is due to protein phosphorylation, as treatment with calf intestinal alkaline phosphatase (CIP) converts most M ek 1 to the faster migrating form (Fig. 5B). Only the faster migrating form of M ek1 is present in the mek1-D290A mutant, suggesting that $M$ ek 1 kinase activity is required for $M$ ek 1 phosphorylation in vivo.

\section{Red1 displays Mek1-dependent phosphorylation}

Because $M$ ek 1 exhibits protein kinase activity and M ek 1 interacts with Red1 and Hop1, Mek1-dependent phosphorylation of Red1 and Hopl was tested. When Red1 and Hopl are precipitated from wild-type meiotic cell extracts with antibodies to Redl or to Hopl, respectively, these proteins undergo phosphorylation in vitro (Fig. 6A,B). This phosphorylation depends on $M$ ek 1 , as little or no phosphorylation of Redl or Hopl is observed in immunoprecipitates from mek1 mutants. Mek1-dependent phosphorylation of Redl and Hopl in vitro is also observed if anti-M ek1 antibodies are used to immunoprecipitate Red1 and Hopl (data not shown).

Table 1. Two- and three-hybrid protein interactions

\begin{tabular}{|c|c|c|c|c|c|}
\hline \multirow[b]{2}{*}{ Strains } & \multirow{2}{*}{$\begin{array}{l}\text { DNA-binding } \\
\text { domain }^{a}\end{array}$} & \multirow{2}{*}{$\begin{array}{l}\text { Activation } \\
\text { domain }\end{array}$} & \multirow[b]{2}{*}{ Overproduced } & \multicolumn{2}{|c|}{$\beta-G$ alactosi dase activity } \\
\hline & & & & vegetative & meiotic \\
\hline Wild type (JM 239) & M ek1 aa 1-497 (pJ54) & Red1 (pJ63) & & 30.0 & 123.0 \\
\hline Wild type (JM 239) & M ek1 aa 1-100 (pJ61) & Red1 (pj63) & & 26.8 & 91.6 \\
\hline Wild type (JM 239) & M ek1 aa 1-63 (pJ71) & Red1 (pJ63) & & 30.5 & 91.5 \\
\hline Wild type (JM 239) & M ek1 aa 1-497 (pJ54) & Hop1 (pJ64) & & 1.3 & 93.6 \\
\hline red1::U RA3 (JM 240) & M ek1 aa 1-497 (pJ54) & Hop1 (pJ64) & & N.D. & 0.2 \\
\hline Wild type (JM 239) & M ek1 aa 1-497 (pJ54) & Hop1 (pJ64) & Red1 (pJ89) & 42.0 & 93.1 \\
\hline Wild type (JM 239) & M ek1 aa 1-497 (pJ54) & vector (pACTII) & & 1.0 & 0 \\
\hline Wild type (JM 239) & M ek1 aa 1-497 (pJ54) & vector (pACTII) & Red1 (pJ89) & 1.1 & 0 \\
\hline Wild type (JM 239) & vector (pBG 4D-1) & Red1 (pj63) & & 1.2 & 0 \\
\hline Wild type (JM 239) & vector (pBG4D-1) & Hopl (pJ64) & & 1.2 & 0 \\
\hline
\end{tabular}

N umbers shown are averages obtained from triplicate cultures that were assayed for vegetative and meiotic $\beta$-galactosidase activity, measured as moles o-nitrophenyl- $\beta$-D-gal actosi de hydrolyzed per minute per milligram of protein (Durfee et al. 1993). N ames of yeast strains and plasmids are indicated in parentheses. (N.D.) N ot determined.

a(aa) Amino acids. 


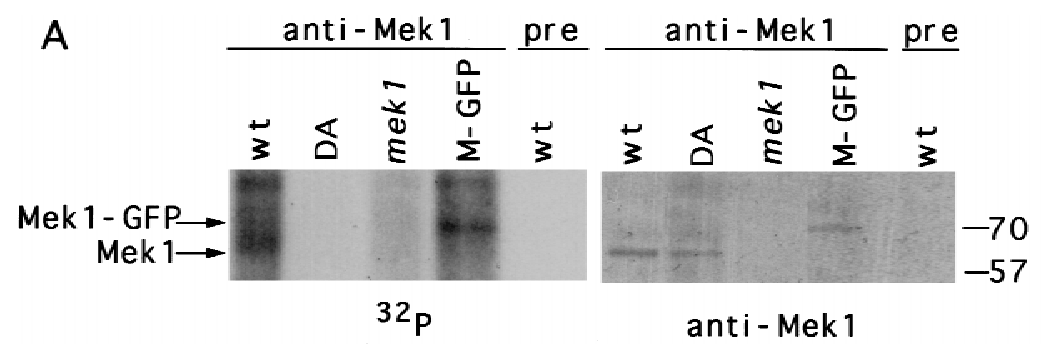

B

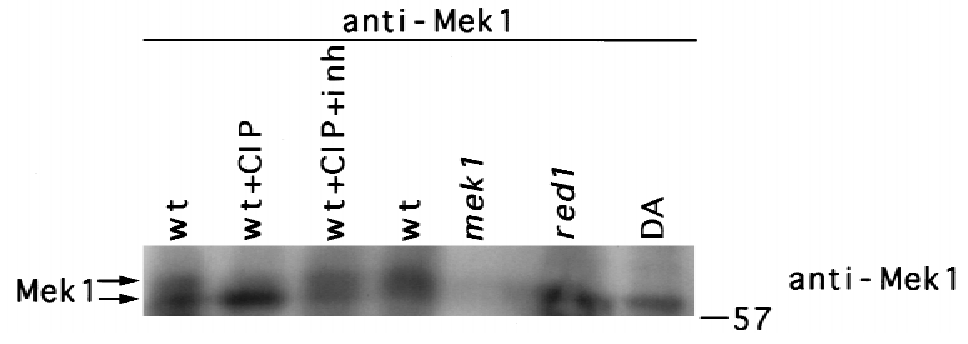

Figure 5. Mek 1 is a phosphoprotein that displays kinase activity. (A) Mek1 kinase activity in vitro. Anti-Mek1 immunoprecipitates were incubated with $\left[\gamma^{-32}\right.$ P]ATP, separated by an $8 \%$ SDS-polyacrylamide gel, and analyzed by autoradiography and immunoblotting with anti-M ek1 antibodies. (B) Gel mobility shift analysis of M ek1. Anti-Mek1 immunoprecipitates were untreated, treated with CIP, or treated with CIP plus phosphatase inhibitor (CIP + inh). Conditions of electrophoresis ( $20 \mathrm{~V}$ for 16 $\mathrm{hr}$ ) were slower than those used in A. The Mek1 protein was detected by immunoblotting. M olecular mass markers (in kD) are at right. Strains used are wild type (BR2495), mek1-D290A (JM 191), mek1:: LYS2 (JM 82), MEK1::GFP (JM 98), and red1 (MY231). (wt) Wild type; (DA) mek1D290A; (M-GFP) Mek1-GFP; (pre) preimmune serum.
To confirm that the proteins phosphorylated in vitro are indeed Red1 and Hopl, these proteins were tagged with a hemaggl utinin (HA) epitope to generate Red1-HA and Hopl-HA fusion proteins. When Redl is immunoprecipitated from a strain heterozygous for the RED 1HA fusion gene, both the band presumed to represent Red1 and an additional band of slightly greater mol ecular mass are observed on the autoradiograph and on the immunobl ot. This extra band is recognized by both anti-HA antibodies and anti-Redl antibodies, indicating that it is the Red1-HA protein (data not shown). Similar results were obtained with Hopl-HA (data not shown).

Gel mobility shift assays were employed to assess Red1 phosphorylation in vivo. When Red1 protein is immunoprecipitated from meiotic cell extracts using antiRed1 antibodies, two mobility forms of Red1 are observed (Fig. 6C). The slower migrating form is due to phosphorylation, as treatment with CIP converts much of the Redl protein to the faster migrating form (Fig. 6C). In mek 1 mutants, only the faster mi grating form of Red 1 is observed (Fig. 6C). Thus, Red1 is a phosphoprotein that displays M ek1-dependent phosphorylation in vivo.

Although M ek1-dependent phosphorylation of Hopl is observed in vitro, no obvious gel mobility shift of Hop1 was detected in vivo (data not shown). It is possible that Hopl is nonspecifically phosphorylated in vitro but this phosphorylation is not biologically relevant.

Mek 1 kinase activity is required

for sister-chromatid cohesion

One function proposed for Red1 is meiotic sister-chromatid cohesion (Rockmill and Roeder 1990; Smith and Roeder 1997). To determine whether this is the case and whether M ek1 contributes to sister-chromatid cohesion, precocious separation of sister chromatids was analyzed on spread pachytene chromosomes by fluorescent in situ hybridization (FISH) (Table 2).
In a wild-type pachytene nucleus, chromosomes are homologously paired and sister chromatids are tightly associated, resulting in a single FISH signal (class A). The presence of a doublet (class B) indicates either that homologs are paired, but not yet fused, or that homologs have started to separate during meiosis I. During and after the meiosis I division, but before meiosis II, two separate signals (class C) are observed. Class C may al so represent prophase nuclei in which homologs have failed to pair. M utants that display defects in homolog pairing, such as red1 and hop1 ( $\mathrm{N}$ ag et al. 1995), are expected to show an increase in class $\mathrm{C}$ nuclei, even if sister chromatids remain tightly associated throughout meiosis I.

T wo novel classes of FISH si gnals are observed in red1 and mek 1 mutants and rarely in hopl. Class D nuclei exhibit a doublet plus an additional signal, whereas class E nuclei contain three separate signals. In both classes, one pair of sister chromatids has separated precociously. The increase in class D and E nuclei in mek 1 and red1 indicates that these mutants suffer defects in sister-chromatid cohesion (Table 2 ). The mek 1 red 1 double mutant displays a defect in cohesion similar in magnitude to each of the single mutants, indicating that Mek1 and Red1 affect sister-chromatid cohesion in the same way. These observations raise the possibility that Mek1-dependent phosphorylation of Redl establishes meiotic sister-chromatid cohesion.

\section{Discussion}

Mek1 interacts with components of meiotic chromosomes

We have demonstrated that the M ek 1 kinase localizes to meiotic chromosomes and specifically promotes morphogenesis of the SC and meiotic sister-chromatid cohesion. The colocalization of M ek1 with Redl and the dependency of M ek1 localization on both Red1 and Hop1 
A

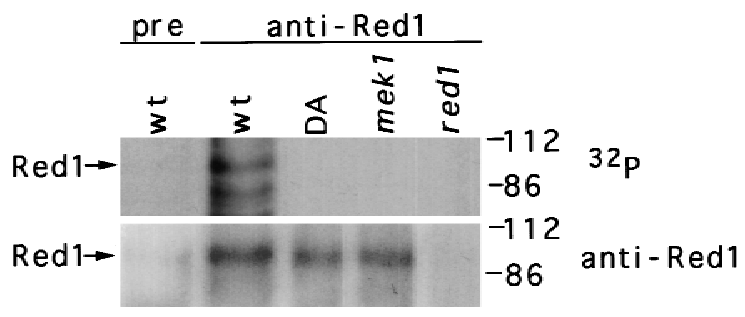

B

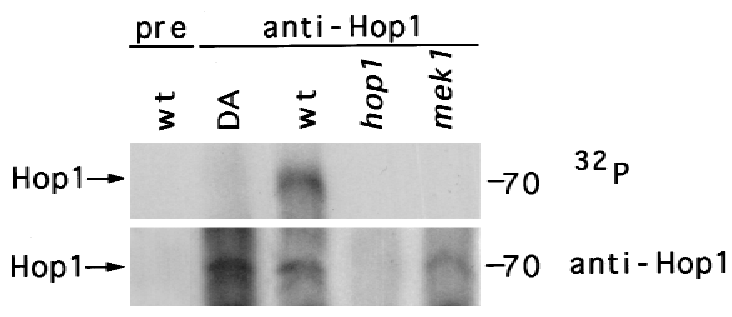

C

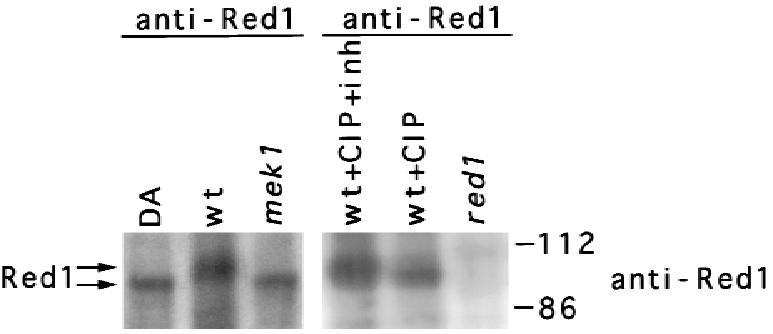

Figure 6. Mek1-dependent phosphorylation of Red1. (A) In vitro phosphorylation of Red1 protein immunoprecipitated with anti-Red1 antibodies. (B) In vitro phosphorylation of Hopl immunoprecipitated with antibodies to Hopl. Immunoprecipitates were incubated with $\left[\gamma_{-}{ }^{32} \mathrm{P}\right] \mathrm{ATP}$, then analyzed by SDSPAGE, autoradiography, and immunoblotting with antibodies to either Red1 (A) or Hopl (B). Additional phosphorylated bands may represent other proteins in the immune complex. (C) Gel mobility shift analysis of Red1. Anti-Red1 immunoprecipitates were untreated, treated with CIP, or treated with CIP plus inhibitor (CIP +inh). Immunoprecipitates were analyzed by sl ower conditions of el ectrophoresis ( $20 \mathrm{~V}$ for $16 \mathrm{hr}$ ) than used in A. The Redl protein was detected by immunoblotting. M olecular mass markers (in kD) are at right. Strains used are wild type (BR2495), mek1-D290A (JM 191), mek1::LYS2 (JM 82), and red1::U RA 3 (MY231). (wt) Wild type; (DA) mek1-D290A; (pre) preimmune serum.

imply that $\mathrm{M}$ ek 1 associates with axial elements and SC lateral elements. Mek1 chromosomal localization, like that of Red1 and Hopl, does not depend on the initiation of meiotic recombination or the formation of mature SC (J.M. Bailis and G.S. Roeder, unpubl.).

M ek1 and Red1 can associate in the absence of Hopl in the two-hybrid system, and Mek1 and Red1 both localize to the nucleolus, where Hopl is not present (Smith and Roeder 1997; unpubl.). Also, the hopl mutant displays only a minor defect in sister-chromatid cohesion, as expected if M ek1-dependent phosphorylation of Red1 is important for cohesion, and M ek 1 and Redl can interact in the absence of Hopl. Two observations, however, suggest that Hopl normally stabilizes the associa- tion of Mek1 with Red1. First, Mek1 localization to chromosomes is detected in only a small fraction of nuclei from the hop1 mutant. Second, anti-M ek1 antibodies appear to precipitate less Red1 protein from a hop1 mutant than from wild type (J.M. Bailis and G.S. Roeder, unpubl.).

Interaction between M ek 1 and Hopl is detected only if Red1 is also present. Hopl, like M ek1, is dependent on Red1 for its localization to chromosomes (Smith and Roeder 1997). The localization requirements of Hopl and M ek1 support a specific order of protein assembly: Red1, then Hopl, and finally Mek1. These proteins may associate with each other on chromosomes, or they may first assemble into a complex that then local izes to chromosomes. The order of protein assembly is consistent with the severity of the SC defects observed in the mutants. red 1 fails to form any SC or axial el ements (Rockmill and Roeder 1990), whereas hopl forms axial elements that do not synapse (Hollingsworth and Byers 1989; Loidl et al. 1994). mek1 undergoes extensive SC formation (Rockmill and Roeder 1991).

Experiments presented here indicate that the first 63 amino acids of $M$ ek 1 are sufficient to interact with Red1. The Mek1 amino terminus contains a forkhead-associated homology domain (FHA) (amino acids 47-102; Hofmann and Bucher 1995). FHA domains are al so present in the yeast mitotic protein kinases Rad53 and Dun1 (Zhou and Elledge 1993; Sun et al. 1996, 1998); the interaction of the FHA 2 domain of Rad53 with Rad9 (Sun et al. 1998) suggests that this motif serves as a protein-binding domain. However, the first 63 amino acids of $M$ ek 1 do not contain the whole FHA domain; only one of three conserved sequence motifs within the FHA domain is pre sent in this region of Mek1.

Much as Rad53 and Dun1 are involved in sensing DN A damage and transducing signals during vegetative growth (Zhou and Elledge 1993; Sun et al. 1996), the interaction between Mek1 and meiotic chromosomal proteins may function to sense and/or transduce signals about the progress of synapsis and/or meiotic recombination (Xu et al. 1997). Consistent with this hypothesis, a red1 or a mek 1 mutation restores sporulation to mutants that arrest at pachytene due to a checkpoint triggered by defects in recombination and/ or synapsis (Xu et al. 1997). M ek1 kinase function is required to effect this pachytene checkpoint, as the mek1-D290A mutation also restores sporulation to zip1 (J.M. Bailis and G.S. Roeder, unpubl.). Xu et al. (1997) have suggested that the chromosomal context of M ek 1 and Red1 is important for monitoring mei otic recombination; this context may be established through M ek1-dependent phosphorylation of Red1.

Hollingsworth and Ponte (1997) have proposed that phosphorylation of Hopl or Red1 by Mek1 promotes SC assembly. In a specific non-null mek1 mutant (mek1974), overproduction of Red1 decreases recombination and viability, whereas overproduction of Hopl improves recombination and viability. These observations led to the speculation that there is an excess of Red1 rel ative to Hopl on chromosomes in the mek 1 mutant compared to 
Table 2. FISH analysis of meiotic sister-chromatid cohesion

\begin{tabular}{|c|c|c|c|c|c|c|}
\hline Strain & $\begin{array}{c}\text { Class A } \\
1 \\
0\end{array}$ & $\begin{array}{l}\text { Class B } \\
\text { doublet }\end{array}$ & $\begin{array}{c}\text { Class C } \\
2 \\
\end{array}$ & $\begin{array}{c}\text { Class D } \\
\text { doublet }+1 \\
\end{array}$ & $\begin{array}{c}\text { Class E } \\
3\end{array}$ & Percent PSSC \\
\hline Wild type (BR2495) & 74.0 & 13.0 & 13.0 & 0 & 0 & 0 \\
\hline mek $1 \Delta$ (JM 82) & 48.0 & 14.0 & 19.0 & 13.0 & 6.0 & 19.0 \\
\hline mek1-D290A (JM 191) & 49.0 & 17.0 & 17.5 & 15.0 & 2.0 & 17.0 \\
\hline red1s (MY231) & 38.5 & 8.5 & 31.5 & 11.0 & 10.5 & 21.5 \\
\hline hop1s (BR2498) & 49.0 & 12.0 & 36.0 & 2.0 & 1.0 & 3.0 \\
\hline mek $1 \Delta$ red $1 \Delta$ (JM 235) & 56.5 & 9.0 & 16.0 & 11.0 & 7.5 & 18.5 \\
\hline
\end{tabular}

Spread meiotic nuclei were prepared after $15 \mathrm{hr}$ in sporulation medium and then hybridized with a probe for chromosome III. Two hundred nuclei were scored for the number and position of condensed FISH signals; the percentage observed for each class is shown (see text for explanation). The percentage of precocious separation of sister chromatids (PSSC) was cal culated as the sum of class D and class $\mathrm{E}$ nuclei divided by the total number of nuclei scored.

wild type. This hypothesis is inconsistent with our observation that Hopl appears to assemble onto chromosomes normally in the absence of Mek1 but does not dissociate efficiently. However, our observations can be reconciled with those of Hollingsworth and Ponte (1997). We have found that Red1 overproduction decreases recombination in wild type and in a zipl mutant and al so decreases spore viability in zipl (J.M. Bailis, A.V. Smith, and G.S. Roeder, in prep.), which strongly suggests that the effect of Red1 overproduction observed by Hollingsworth and Ponte (1997) is not specific to the mek1-974 mutant. If the mek1-974 mutant exhibits decreased Mek1 kinase activity, then overproduction of Hopl might create more opportunities for M ek1 to phosphorylate Red1 and/or Hopl by increasing the number of Red1-Hopl complexes available as substrates. Alternatively, the M ek1-974 mutant protein might be defective in interacting with Red1 and/or Hopl, and overproduction of Hopl might promote M ek1 binding by increasing the number of Red1-Hopl complexes available.

\section{Mek1 function depends on its kinase activity}

M ek1 protein kinase activity is necessary for its mei otic function, as the mek1-D290A mutant displays a reduction in spore viability equivalent to that of the mek 1 null mutant. The simplest explanation of these resul ts is that Mek1 directly phosphorylates Red1; however, an indirect interaction cannot be ruled out. It is possible that other protein kinases coprecipitate with M ek1 and that M ek1, like its mitotic counterparts, functions as part of a protein kinase cascade. Also, it is not yet clear what signal serves to trigger Mek1 phosphorylation/activation, or whether this signaling requires other protein kinases. In many cases, kinases require phosphorylation or autophosphorylation to translocate to the nucleus (for review, see Jans 1995). However, M ek1 local izes to chromosomes in both the mek1-lacZ and mek1-D290A mutants (which lack kinase activity), suggesting that phosphorylation of Mek1 (and Red1) need not occur until after M ek 1 becomes associated with chromosomes. The fact that Mek 1 is not phosphorylated in a red1 mutant (J.M. Bailis and G.S. Roeder, unpubl.) is consistent with this hypothesis.
Mek1 may promote Hopl dissociation from chromosomes

Hopl remains associated with chromosomes longer in mek 1 mutants than in wild type, suggesting that M ek 1 kinase activity aids in Hopl dissociation from meiotic chromosomes, an event that is correlated with the production of mature SC (Smith and Roeder 1997). Because Hopl is required to promote interhomolog recombination (Hollingsworth and Byers 1989; Schwacha and Kleckner 1994), and Hopl demonstrates DNA-binding capability (Hollingsworth et al. 1990; Kironmai et al. 1998), it is possible that Hopl directly associates with DNA undergoing recombination. The interaction of Mek1 with Redl and/or Hopl might release Hopl from the DN A, allowing recombi nation events to proceed and synapsis to be completed. This interpretation is consistent with evidence that $\mathrm{M}$ ek 1 monitors mei otic recombination (Xu et al. 1997) and that Hopl di ssociation from DNA is slow in an vitro assay from which Mek1 is absent (Kironmai et al. 1998). However, Hopl does eventually dissociate from chromosomes in the absence of M ek1, indicating that M ek 1 kinase activity is not a prerequisite for $\mathrm{Hopl}$ release from chromosomes.

Mek1-dependent phosphorylation of Red1 may establish meiotic sister-chromatid cohesion

FISH analyses of precocious separation of sister chromatids provide evidence that Redl plays a role in meiotic sister-chromatid cohesion and suggests that phosphorylation of Red1 by Mek1 regulates this function. M eiotic sister-chromatid cohesion may involve the phosphorylation of SC components in organisms other than yeast. The hamster Corl protein is a lateral element component that is presumed to play a role in sister-chromatid cohesion (M oens and Spyropoulos 1995), and the rat homolog of Cor1, SCP3, has been shown to be a phosphoprotein (Lammers et al . 1994). A dditional ly, two forms of the yeast $\mathrm{Mcdl} / \mathrm{Sccl}$ protein are detected by Western bl ots, suggesting that regulation of mitotic cohesion may also involve protein phosphorylation (Guacci et al. 1997).

It is interesting to note that a defect in sister-chroma- 
tid cohesion is associated with a decrease in mei otic recombination, suggesting a functional relationship between cohesion and recombination. In the redl and mek 1 mutants, recombination is reduced to $20 \%$ of the wild-type level (Rockmill and Roeder 1990, 1991; Leem and Ogawa 1992). Drosophila ord mutants and Schizosaccharomyces pombe rec8 mutants also di splay defects in both recombination and meiotic sister-chromatid cohesion (Miyazaki and Orr-Weaver 1994; Molnar et al. 1995). Recombination might be reduced if cells fail to discriminate sisters from nonsisters and therefore fail to favor interhomolog (i.e., nonsister) interactions (Smith and Roeder 1997). Alternatively, failure to establish sister-chromatid cohesion may result in chromosomal axes that are developed improperly and therefore serve as less suitable substrates for the initiation of interhomolog recombination (Schwacha and Kleckner 1997; Xu et al. 1997). On the other hand, it is possible that recombination contributes to cohesion. Consistent with this possibility, insertion of a recombination hot spot into a yeast artificial chromosome enhances sister-chromatid cohesion (Sears et al. 1994). Also, a defect in sister-chromatid cohesion is observed in the dmcl mutant (Rockmill and Roeder 1994), which lacks a meiosis-specific homolog of the RecA strand exchange enzyme (Bishop et al. 1992).

Our proposal that Mek1 functions in meiotic sisterchromatid cohesion may al so explain why the mek 1 mutant forms short stretches of SC instead of full-length complexes (Rockmill and Roeder 1991). In the spo76 mutant of Sordaria (M oreau et al . 1985), extensi ve SC forms, but there are regions where axial elements are unsynapsed. These axial elements are often split into two, thinner elements; however, lateral el ements within SC never appear to be subdivided. These observations suggest that Spo76 functions in sister-chromatid cohesion and that synapsis cannot occur in regions where sisters are not properly associated (Moreau et al. 1985). Thus, the interruptions in SC observed in the mek1 mutant may correspond to regi ons where meiotic sister-chromatid cohesion has failed.

\section{Materials and methods}

\section{Plasmids}

Standard methods were used in plasmid constructions (Sambrook et al. 1989). pB121 carries the null mek1::LYS2 mutation (Rockmill and Roeder 1991). pJ68, constructed by $\mathrm{K}$. $\mathrm{N}$ andabalan (Yale University, New Haven, CT), contains the Spel-Pvull fragment of MEK 1 inserted at the Xbal site of pGEXKG (Guan and Dixon 1991), such that MEK1 is downstream of glutathione S-transferase and fused in-frame (GST-Mek1). pR1284 carries an in-frame fusion of the lacZ gene inserted after 189 nucleotides of the MEK1 coding region (Burns et al. 1994).

The mek1-D290A mutation was constructed by PCR using the method of Zhou and Elledge (1993). The Spel-H pal region of MEK 1 containing this mutation was substituted into the Spel$\mathrm{H}$ pal sites of pJ77, which contains the EcoRI-Pvull fragment of MEK1 inserted into the EcoRI-EcoRV sites of YIp5 (Rothstein 1991). The resulting plasmid, pJ30, contains the mek1-D290A mutation as confirmed by sequencing.
An in-frame fusion of MEK1 with GFP was constructed as follows. PCR was used to generate a N otl site immediately preceding the MEK1 stop codon and a Sall site immediately following the stop codon. An Hpal-Sall fragment of MEKI (containing the $\mathrm{N}$ otl site) was cl oned into pB124, which contains the MEK1 EcoRI-Pvull fragment inserted at the EcoRI-EcoRV sites of pBR322 (Rockmill and Roeder 1991). Then, the Notl (filled in)-Xbal fragment of MEK1 was inserted into the $\mathrm{Nhel}$ (filled in)-Sall sites of a multicopy plasmid containing the GFP gene (pCB431; Chua and Roeder 1998), creating pJ 23.

For plasmids used in the two-hybrid assay, the RED1-coding region was amplified such that a BamHI site was introduced immediately preceding the start codon, and a Sall site was introduced directly before the stop codon. PCR was used to generate a Bglll site in HOP1 just before the start codon and a Sall site immediately before the stop codon. pJ 63 contains the RED 1 PCR product (cut with BamHI and SalI) cloned into the BamHIXhol sites of pACTII, which contains the GAL4 activation domain (Bai and Elledge 1997). The HOP1 PCR product was cut with BglII and Sall and inserted into the BamHI-Sall sites of pACTII to generate pJ64. The MEK1-coding region was amplified such that a BamHI site was introduced before the start codon and a Sall site was generated before the stop codon; this PCR product was cut with BamHI and Sall and ligated into the BamHI-Sall sites of pBG 4D-1, which contains the GAL4 DNAbinding domain (Durfee et al. 1993), to produce pJ54. pJ61 encodes the first 100 codons of $\mathrm{M} \mathrm{ek} 1$ (on a BamHI-Spel fragment of pJ54) in the BamHI-Xbal sites of pBG4D-1. A MEK1 fragment encoding the first 63 amino acids of Mek1 was generated by PCR, cut with BamHI and Sall (the Sall site was introduced by PCR), and cloned into the BamHI-Sall sites of pBG 4D-1 to generate pJ71. The Nael-Aatll fragment containing HIS3 from pRS423 (Sikorski and Hieter 1989) was cloned into the PmllAatll sites of pYADE4 (Brunelli and Pall 1993), producing pJ 88. Then, to generate pJ89, which contains RED1 downstream of the ADH1 promoter, the BamHI-Sall RED1 PCR product (above) was inserted into pJ 88 at the BamHI-Sall sites.

\section{Yeast strains}

Yeast strains were constructed and maintained with standard procedures and media (Sherman et al. 1986). Transformation of yeast was carried out by the lithium acetate procedure of Gietz et al. (1995). Gene disruptions were confirmed by Southern bl ot analysis.

All strains used for cytology and for immunoprecipitations are isogenic with BR2495 (Rockmill and Roeder 1990). Homozygous mutant red1::U RA 3 and hop1::TRP1 strains are MY231 and BR2498, respectively (Smith and Roeder 1997). Homozygous mutant mek 1::LYS2 (JM 82) and mek1-lacZ (JM 198) strains were constructed using pB121 and pR1284, respectively. JM 191 (homozygous for mek1-D290A) was generated by two-step transplacement (Rothstein 1991) using pJ30. The homozygous mek1::LYS2 red1::URA3 double mutant (JM235) was constructed using pB121, and then pV180 (Smith and Roeder 1997). The haploid parents of JM 198 were transformed with either pNH32-1 (hopl::TRP1; Hollingsworth and Byers 1989) or pV180, and then mated to produce the homozygous mutant mek1-lacZ red1::URA3 (JM93) and mek1-lacZ hop1::TRP1 (JM 88) diploids. Strain JM 98 (MEK1::GFP) is JM 82 carrying pJ 23.

Strains containing the Red1-HA and Hopl-HA fusion proteins were constructed by PCR as described (Schneider et al. 1995). The PCR products were transformed into yeast, and correct integration was confirmed by PCR and immunoblotting. Strains JM 241 and JM 142 are BR2495 that is heterozygous for Red1-HA or Hop1-HA, respectively. 
The yeast strain used for the two-hybrid protein system is JM 239, a diploid constructed by inducing mating type switching of PJ69-4A (James et al. 1996) and mating MATa and MAT $\alpha$ haploid strains. The red1::URA3 mutant version of JM 239 (JM 240) was constructed using pV180.

\section{Mek1 antibodies}

GST-M ek1 protein was purified from Escherichia coli containing pJ 68 by the methods of Guan and Dixon (1991). GST-M ek1 was then used to raise antibodies in rabbits and mice at the Pocono Rabbit Farm and Laboratory (Canadensis, PA).

\section{Cytology}

M eiotic chromosomes were spread and stained with antibodies as described (Chua and Roeder 1998). Anti-Mek1 antibodies were affinity purified from serum (Chua and Roeder 1998) and used at a 1:40 dilution. Anti-rabbit antibodies conjugated to biotin, or anti-mouse antibodies conjugated to biotin (Zymed Laboratories), were used at a 1:200 dilution as secondary antibodies. Antibodies labeled with streptavidin conjugated to Texas red (Jackson ImmunoResearch Laboratories) were used at a 1:200 dilution to detect primary and secondary antibodies (Page and Snyder 1992).

Anti-Redl and anti-Hopl antibodies (Smith and Roeder 1997), as well as anti-Zipl antibodies (Sym et al. 1993), were used at 1:100 dilution. Anti- $\beta$-galactosi dase antibodies (ICN Biochemicals) were used at 1:40 dilution. Antibodies other than antiMekl antibodies were detected using rabbit or mouse antibodies conjugated to Oregon green (Molecular Probes). Chromosomal DNA was stained with $1 \mu \mathrm{g} / \mathrm{ml}$ DAPI. Images were recorded and analyzed using a Leitz DMRB microscope and a Photometrics Imagepoint CCD camera.

To determine the amount of overlap between Mek1, Red1, and Hopl, the total number of M ek1 foci per spread nucleus per time point were compared with the number of M ek 1 foci that al so contain Red1 or Hopl. M ek1 overlap with Red1 was examined for at least 200 nuclei. Mek1 overlap with Hopl was assessed for 200 nuclei both in wild type and in the mek1-D290A mutant. Fortuitous colocalization was assessed by rotating an overlay corresponding to one of the two signals by $90^{\circ} \mathrm{C}$ or $180^{\circ} \mathrm{C}$ and determining the amount of overlap between the misoriented images, as described by Gasior et al. (1998).

\section{Immunoprecipitations and Western blots}

To prepare meiotic cell extracts, cells were grown to saturation in one liter of YPD (Sherman et al. 1986) supplemented with 60 $\mu \mathrm{g} / \mathrm{ml}$ uracil and $40 \mu \mathrm{g} / \mathrm{ml}$ adenine; cells were then pelleted and resuspended in 3 liters of sporulation medium ( $2 \%$ potassium acetate). After 13-14 hr, cells were collected by centrifugation and resuspended in $3 \mathrm{ml}$ of lysis buffer $(50 \mathrm{~mm}$ Tris, $250 \mathrm{~mm}$ $\mathrm{NaCl}, 5 \mathrm{~mm}$ EDTA, $50 \mathrm{~mm} \mathrm{NaF}, 0.1 \% \mathrm{~N} \mathrm{P}-40$ at pH 7.8) to which protease inhibitors $(100 \mu \mathrm{g} / \mathrm{ml}$ PMSF, $20 \mu \mathrm{g} / \mathrm{ml}$ aprotinin, 10 $\mu \mathrm{g} / \mathrm{ml}$ antipain, $10 \mu \mathrm{g} / \mathrm{ml}$ leupeptin, $10 \mu \mathrm{g} / \mathrm{ml}$ pepstatin $A$; Sigma) and $1.5 \mathrm{~mm}$ dithiothreitol (DTT) were added. Glass beads (425-600 $\mu \mathrm{m})$ (Biospec Products; Sigma) were added to threefourths of the original volume. Cell lysis was accomplished by vortexing at $4^{\circ} \mathrm{C}$ for 15 intervals of 1 min each, with tubes incubated on ice for 3-5 min between periods of vortexing. After centrifugation at 15,000 rpm in a SS-34 Sorvall rotor for $20 \mathrm{~min}$ at $4^{\circ} \mathrm{C}$, supernatants (containing at least $50 \mathrm{mg}$ of total protein) were recovered and used for immunoprecipitations.
Immunoprecipitations were carried out at $4^{\circ} \mathrm{C}$ on a nutator (A dams/ALA Scientific Instruments). Anti-M ek1 serum (1:200), anti-Red1 serum (1:500), anti-Hopl serum (1:500), anti-ß-gal actosidase antibody (1:500), or preimmune serum (1:500) was added to each supernatant. After $2 \mathrm{hr}, 40 \mu \mathrm{l}$ of Protein A-Sepharose (Pierce) was added, and incubations were allowed to proceed for an additional hour. At the start of immunoprecipitations, protease inhibitors were added to final concentrations twice that originally included for cell Iysis. Immune complexes were collected by centrifugation, washed twice in IP buffer (20 $\mathrm{mm}$ Tris, $100 \mathrm{~mm} \mathrm{~N} \mathrm{aCl}, 2 \mathrm{~mm}$ EDTA, 0.05\% Tween 20 at $\mathrm{pH}$ $7.8)$, boiled in $6 \times$ sample buffer $(70 \%$ Tris- $\mathrm{Cl}$ at $\mathrm{pH} 6.8,30 \%$ glycerol, 10\% SDS, $0.09 \%$ DTT, $0.01 \%$ bromophenol blue), and fractionated by an $8 \%$ SDS-polyacrylamide gel at $100 \mathrm{~V}$ for 3-4 hr (Figs. 4, 5A, and 6A,B) or at $20 \mathrm{~V}$ for $16 \mathrm{hr}$ (Fig. 5B and 6C).

Gels were blotted onto nitrocellulose (Schleicher \& Schuell) using the Mini-Protean II system (Bio-Rad). For Western blot anal ysis (T owbin et al. 1979), filters were incubated in blocking buffer (5\% dry milk and 0.1\% Tween 20 in Tris-buffered saline; Sambrook et al. 1989). After 1 hour, primary antibody was added to a final dilution of 1:200-1:500. Primary antibody was detected using anti-rabbit antibody conjugated to alkaline phosphatase (Jackson ImmunoResearch Laboratories) at a 1:200 dilution, followed by CDP-Star (Boehringer Mannheim). Blots were exposed to Biomax film (Kodak). The Red1 protein migrates at $100 \mathrm{kD}$, as predicted based on sequence; Hopl migrates as a 70-kD protein, as described (Hollingsworth et al. 1990).

\section{Kinase assays}

For in vitro kinase assays, immune complexes were resuspended in $75 \mu \mathrm{l}$ kinase buffer $\left(50 \mathrm{~mm}\right.$ Tris, $10 \mathrm{~mm} \mathrm{M} \mathrm{gCl}_{2}$ at $\mathrm{pH}$ 7.0) after two washes in IP buffer. Nonradioactive ATP was added to $1 \mu \mathrm{m} ; 20 \mu \mathrm{Ci}$ of $\left[\gamma^{-32}{ }^{32}\right]$ ATP (sp. act. $3000 \mathrm{Ci} / \mathrm{mmole}$, Amersham) was added and reactions were allowed to proceed for $20 \mathrm{~min}$ at room temperature. SDS-PAGE and blotting to nitrocellulose were carried out as described above. Filters were exposed to film overnight to 2 days to assess [ $\gamma^{32}$ P]ATP incorporation.

For gel mobility shift analysis, immune complexes were resuspended in $75 \mu \mathrm{l}$ phosphatase buffer $(50 \mathrm{~mm}$ Tris, $20 \mathrm{~mm}$ $\mathrm{M} \mathrm{gCl}_{2}, 40 \mathrm{~mm} \mathrm{KCl}$ at pH 8.0) containing $100 \mu \mathrm{g} / \mathrm{ml}$ PM SF. Sixty units of CIP (Boehringer Mannheim) and $14 \mu$ of $10 \times$ phosphatase buffer (Boehringer $M$ annheim) were added to two samples from wild-type immunoprecipitates; $5 \mathrm{~mm} \beta$-glycerophosphate, a phosphatase inhibitor (Sigma), was al so added to one of these samples. Samples were incubated at $30^{\circ} \mathrm{C}$ for $10 \mathrm{~min}$, and proteins were then analyzed as described above.

\section{Two-hybrid protein assays}

JM 239 derivatives carrying the various combinations of MEK1 and RED1 or MEK1 and HOP1 plasmids (see Table 1) were grown to saturation in $2 \times$ synthetic complete medium lacking leucine and tryptophan. Strain JM 239 carrying MEK1-, HOP1-, and RED 1-containing plasmids was grown in $2 \times$ synthetic complete medium (Sherman et al. 1986) lacking leucine, tryptophan, and histidine. One-half of each culture was diluted 1:100, then grown for an additional $12 \mathrm{hr}$ and collected by centrifugation. The other half of each culture was diluted 1:1 in YPAD and grown for $10 \mathrm{hr}$, then transferred to sporulation medium and collected $15 \mathrm{hr}$ later (approximately pachytene). $\beta$-Galactosidase assays were performed on cells in log phase of vegetative 
growth (Durfee et al. 1993) and on meiotic cells (Chua and Roeder 1998).

\section{FISH}

FISH was carried out as described by Chua and Roeder (1998). A 25-kb fragment of Chromosome III was labeled with digoxigenin and then hybridized to spread chromosomes. Antibodies against digoxigenin conjugated to rhodamine were used to detect the chromosome III probe. Chromosomal DNA was stained with DAPI.

\section{Acknowledgments}

We thank Jennifer Fung, Janet N ovak, Beth Rockmill, and Albert Smith for critical comments on the manuscript. We are grateful to Mike Snyder and David Stern for advice on immunoprecipitations. The Howard Hughes Biopolymer/Keck Foundation Biotechnology Resource Laboratory at Yale University provided oligonucleotides and performed sequence analysis. This work was supported by American Cancer Society grant VM-7G to G.S.R. and by the Howard Hughes M edical Institute.

The publication costs of this article were defrayed in part by payment of page charges. This article must therefore be hereby marked 'advertisement' in accordance with 18 USC section 1734 solely to indicate this fact.

\section{References}

Bai, C. and S.J. Elledge. 1997. Gene identification using the yeast two-hybrid system. Methods Enzymol. 283: 141-156.

Bishop, D., D. Park, L. Xu, and N. Kleckner. 1992. DM C1: A meiosis-specific yeast homolog of $E$. coli recA required for recombination, synaptonemal complex formation, and cell cycle progression. Cell 69: 439-456.

Brunelli, J.P. and M.L. Pall. 1993. A series of yeast shuttle vectors for the expression of cDN As and other DN A sequences. Yeast 9: 1299-1308.

Burns, N., B. Grimwade, P.B. Ross-M acdonald, E.-Y. Choi, K. Finberg, G.S. Roeder, and M. Snyder. 1994. Large-scale analysis of gene expression, protein localization and gene disruption in Saccharomyces cerevisiae. Genes \& Dev. 8: 1087-1105.

Chua, P.R. and G.S. Roeder. 1998. Zip2, a meiosis-specific protein required for the initiation of chromosome synapsis. Cell 93: 349-359.

Durfee T., K. Becherer, P.L. Chen, S.H. Yeh, Y. Yang, A.E. Kilburn, W.H. Lee, and S.J. Elledge. 1993. The retinoblastoma protein associates with the protein phosphatase type 1 cataIytic subunit. Genes \& Dev. 7: 555-569.

Flaggs, G., A.W. Plug, K.M. Dunks, K.E. Mundt, J.C. Ford, M.R. Quiggle, E.M. Taylor, C.H. Westphal, T. Ashley, M. F. Hoekstra, and A.M. Carr. 1997. Atm-dependent interactions of a mammalian Chk1 homolog with meiotic chromosomes. Curr. Biol. 7: 977-986.

Friedman, D.B., N.M. Hollingsworth, and B. Byers. 1994. Insertional mutations in the yeast HOP1 gene: Evidence for multimeric assembly in meiosis. Genetics 136: 449-464.

Gasior, S.L., A.K. Wong, Y. Kora, A. Shinohara, and D.K. Bishop. 1998. Rad52 associates with RPA and functions with Rad55 and Rad57 to assemble meiotic recombination complexes. Genes \& Dev. 12: 2208-2221.

Gietz, R.D., R.H. Schiestl, A.R. Willems, and R.A. Woods. 1995. Studies on the transformation of intact yeast cells by the
LiAc/SS-DN A/PEG procedure. Yeast 11: 355-360.

Guacci, V., D. Koshland, and A. Strunnikov. 1997. A direct link between sister chromatid cohesion and chromosome condensation revealed through the analysis of MCD1 in S. cerevisiae. Cell 91: 47-57.

Guan, K. and J.E. Dixon. 1991. Eukaryotic proteins expressed in Escherichia coli: An improved thrombin cleavage and purification procedure of fusion proteins with glutathione S-transferase. Anal. Biochem. 192: 262-267.

H ofmann, K. and P. Bucher. 1995. The FHA domain: A putative nuclear signalling domain found in protein kinases and transcription factors. Trends Biochem. Sci. 20: 347-349.

Hollingsworth, N. and B. Byers. 1989. HOP1: A yeast meiotic pairing gene. Genetics 121: 445-462.

Hollingsworth, N.M. and A.D. Johnson. 1993. A conditional allele of the Saccharomyces cerevisiae HOP1 gene is suppressed by overexpression of two other meiosis-specific genes: RED1 and REC 104. Genetics 133: 785-797.

Hollingsworth, N.M. and L. Ponte. 1997. Genetic interactions between HOP1, RED1 and MEK1 suggest that MEK1 regulates assembly of axial element components during meiosis in the yeast Saccharomyces cerevisiae. Genetics 147: 33-42.

Hollingsworth, N .M., L. Goetsch, and B. Byers. 1990. The HOP1 gene encodes a mei osis-specific component of yeast chromosomes. Cell 61: 73-84.

James, P., J. Halladay, and E.A. Craig. 1996. Genomic libraries and a host strain designed for highly efficient two-hybrid selection in yeast. Genetics 144: 1425-1436.

Jans, D. 1995. The regulation of protein transport to the nucleus. Biochem. J. 311: 705-716.

Keegan, K.S., D.A. Holtzman, A.W. Plug, E.R. Christenson, E.E. Brainerd, G. Flaggs, N.J. Bentley, E.M. Taylor, M.S. Meyn, S.B. Moss, A.M. Carr, T. Ashley, and M.F. Hoekstra. 1996. The Atr and Atm protein kinases associate with different sites al ong meiotically pai ring chromosomes. Genes \& Dev. 10: $2423-2437$.

Kironmai, K.M., K. Muniyappa, D.B. Friedman, N.M. Hollingsworth, and B. Byers. 1998. DN A-binding activities of Hopl protein, a synaptonemal complex component from Saccharomyces cerevisiae. Mol. Cell Biol. 18: 1424-1435.

Lammers, J.H.M., M. van Aalderen, A.H.F.M. Peters, A.A.M. van Pelt, D.G. de Rooij, P. de Boer, H.H. Offenberg, A.J.J. Dietrich, and C. Heyting. 1994. A change in the phosphorylation pattern of the $30000-33000 \mathrm{M}_{\mathrm{r}}$ synaptonemal complex proteins of the rat between early and mid-pachytene. Chromosoma 104: 154-163.

Leem, S.-H. and H. Ogawa. 1992. The MRE4 gene encodes a novel protein kinase homologue required for meiotic recombination in Saccharomyces cerevisiae. Nucleic Acids Res. 20: 449-457.

Loidl, J., F. Klein, and H. Scherthan. 1994. Homologous pairing is reduced but not abolished in asynaptic mutants of yeast. J. Cell Biol. 125: 1191-1200.

Michaelis, C., R. Ciosk, and K. N asmyth. 1997. Cohesins: Chromosomal proteins that prevent premature separation of sister chromatids. Cell 91: 35-45.

Miyazaki, W.Y. and T.L. Orr-Weaver. 1994. Sister-chromatid cohesion in mitosis and meiosis. Annu. Rev. Genet. 28: 167187.

Moens, P.B. and B. Spyropoulos. 1995. Immunocytology of chiasmata and chromosomal disjunction at mouse meiosis. Chromosoma 104: 175-182.

Molnar, M., J. Bahler, M. Sipiczki, and J. Kohli. 1995. The rec8 gene of Schizosaccharomyces pombe is involved in linear el ement formation, chromosome pairing, and sister-chromatid cohesion during meiosis. Genetics 141: 61-73. 
M oreau, P.J.F., D. Zickler, and G. Leblon. 1985. One class of mutants with disturbed centromere cleavage and chromosome pairing in Sordaria macrospora. Mol. \& Gen. Genet. 198: 189-197.

Nag, D.K., H. Scherthan, B. Rockmill, J. Bhargava, and G.S. Roeder. 1995. Heteroduplex DNA formation and homolog pairing in yeast meiotic mutants. Genetics 141: 75-86.

Page, B.D. and M. Snyder. 1992. CIK1: A developmentally regulated spindle pole body-associated protein important for microtubule functions in Saccharomyces cerevisiae. Genes \& Dev. 6: 1414-1429.

Rockmill, B. and G.S. Roeder. 1990. M eiosis in asynaptic yeast. Genetics 126: 563-574.

_-_. 1991. A meiosis-specific protein kinase homolog required for chromosome synapsis and recombination. Genes \& Dev. 5: 2392-2404.

- - . 1994. The yeast med1 mutant undergoes both meiotic homolog nondisjunction and precocious separation of sister chromatids. Genetics 136: 65-74.

Roeder, G.S. 1997. M ei otic chromosomes: It takes two to tango. Genes \& Dev. 11: 2600-2621.

Rothstein, R. 1991. Targeting, disruption, replacement and allele rescue: Integrative DN A transformation in yeast. Methods Enzymol. 194: 281-301.

Sambrook, J., E.F. Fritsch, and T. Maniatis. 1989. Molecular cloning: A laboratory manual (ed. C. Nolan, N. Ford, N. Irwin and $M$. Ferguson). Cold Spring Harbor Laboratory Press, Cold Spring Harbor, NY.

Schneider, B.L., W. Seufert, B. Steiner, Q.H. Yang, and B. Futcher. 1995. Use of polymerase chain reaction epitope tagging in Saccharomyces cerevisiae. Yeast 11: 1265-1274.

Schwacha, A. and N. Kleckner. 1994. Identification of joint molecules that form frequently between homologs but rarely between sister chromatids during yeast meiosis. Cell 90: 1123-1135.

_-_. 1997. Interhomolog bias during meiotic recombination: Meiotic functions promote a highly differentiated interhomolog-only pathway. Cell 90: 1123-1135.

Sears. D.D., P. Hieter, and G. Simchen. 1994. An implanted recombination hot spot stimulates recombination and enhances sister chromatid cohesion of heterologous YACs during yeast meiosis. Genetics 138: 1055-1065.

Sherman, F., G.R. Fink, and J.B. Hicks. 1986. Methods in yeast genetics: A laboratory manual, Cold Spring Harbor Laboratory, Cold Spring Harbor, NY.

Sikorski, R. and P. Hieter. 1989. A system of shuttle vectors and yeast host strains designed for efficient manipulation of DNA in Saccharomyces cerevisiae. Genetics 122: 19-27.

Smith, A.V. and G S. Roeder. 1997. The yeast Red1 protein localizes to the cores of meiotic chromosomes. J. Cell Biol. 136: 957-967.

Sun, Z., D.S. Fay, F. Marini, M. Foiani, and D.F. Stern. 1996. Spk1/Rad53 is regulated by Mec1-dependent protein phosphorylation in DNA replication and damage checkpoint pathways. Genes \& Dev. 10: 395-406.

Sun, Z., J. Hsiao, D.S. Fay, and D.F. Stern. 1998. Rad53 FHA domain associated with phosphorylated Rad9 in response to the DN A damage checkpoint. Science 281: 272-274.

Sym, M. and G.S. Roeder. 1994. Crossover interference is abolished in the absence of a synaptonemal complex protein. Cell 79: 283-292.

Sym, M., J. Engebrecht, and G.S. Roeder. 1993. ZIP1 is a synaptonemal complex protein required for meiotic chromosome synapsis. Cell 72: 365-378.

Towbin, H., T. Staehelin, and J. Gordon. 1979. Electrophoretic transfer of proteins from polyacrylamide gels to nitrocellu- lose sheets: Procedure and some applications. Proc. Natl. Acad. Sci. 76: 4350-4354.

Tu, J., W. Song, and M. Carlson. 1996. Protein phosphatase type 1 interacts with proteins required for meiosis and other celIular processes in Saccharomyces cerevisiae. Mol. Cell. Biol. 16: 4199-4206.

Xu, L., B.M. Weiner, and N. Kleckner. 1997. M eiotic cells monitor the status of the interhomolog recombination complex. Genes \& Dev. 11: 106-118.

Zhou, Z. and S.J. Elledge. 1993. DU N 1 encodes a protein kinase that controls the DNA damage response in yeast. Cell 75: 1119-1127. 


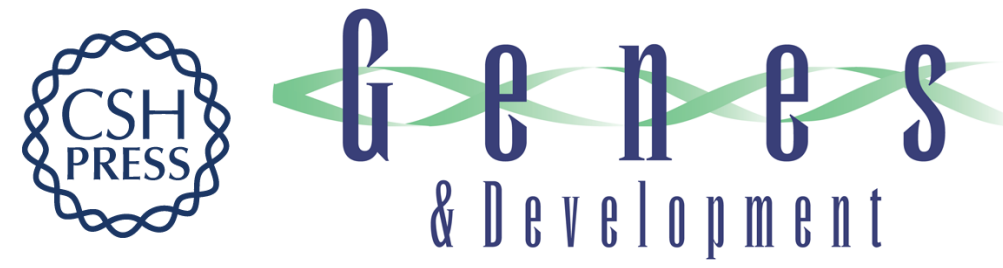

\section{Synaptonemal complex morphogenesis and sister-chromatid cohesion require Mek1-dependent phosphorylation of a meiotic chromosomal protein}

Julie M. Bailis and G. Shirleen Roeder

Genes Dev. 1998, 12:

Access the most recent version at doi:10.1101/gad.12.22.3551

References This article cites 49 articles, 26 of which can be accessed free at:

http://genesdev.cshlp.org/content/12/22/3551.full.html\#ref-list-1

License

Email Alerting Receive free email alerts when new articles cite this article - sign up in the box at the top Service right corner of the article or click here.

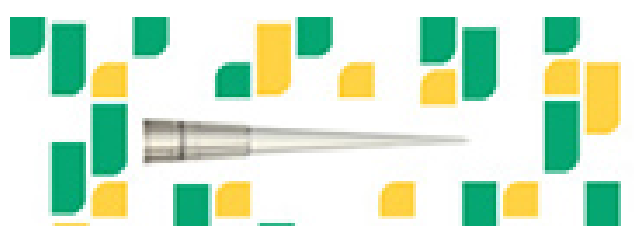

Focused on your science. 